\title{
A Three-Stage Optimization Model for Scheduling the Facilities Maintenance Considering Random Failure Rate
}

\author{
Farank Zagia ${ }^{1}$, Omid Motamedi Sedeh ${ }^{2,}$ Bakhtiar Ostadi ${ }^{3 *}$ \\ ${ }^{1}$ Master of Civil Engineering, Islamic Azad University Center Tehran Branch, Tehran, Iran, \\ ${ }^{2} \mathrm{PhD}$, Faculty of Industrial and Systems Engineering, Tarbiat Modares University, Tehran, Iran
} 3 Associate Professor, Faculty of Industrial and Systems Engineering, Tarbiat Modares University, Tehran, Iran. +989125795141

\begin{abstract}
The increasing value of facilities, on the one hand, and the complexity of the equipment used in them, on the other, have increased the importance of planning for the maintenance of facilities, especially for companies which their facilities are located in different locations. In this paper, a new hybrid model has been presented to optimize facility maintenance scheduling by a combination of Genetic Algorithms (GA), Particle Swarm Optimization (PSO) and the Monte Carlo Simulation for organizing facilities which are in different locations as well as determining the optimum number of crews with three different skills of mechanical, electrical and simple workers. The main contributions of this paper include: (a) optimizing the number of crew by different skills in the first stage. (b) evaluation of fitness value for each solution through the Monte Carlo Simulation Model. (c) scheduling by consideration different failure rates for different facilities in different locations. In order to evaluate the performance of the proposed model, the model has been compared with Golpira's model, the results of which have shown that it is possible to reduce the cost by just over 39\% and reduce MTBF by over half.
\end{abstract}

Key Words: Asset Management, multi locations facilities, Maintenance Scheduling, Heuristic algorithm, Genetic Algorithm

\section{Introduction}

Facility Management (FM) is a process of managing and maintaining facilities in an organization. Facilities can include an office complex, physical resources at the company or site and any other mechanical and electrical utilities that can cause health or create safety hazards for employees. Facility management is an integrated approach for an organization to operate, maintain, improve and adapt its buildings and infrastructures in such a way that the primary objectives of the organization, occupants, owners and facility managers are supported. [1]

Facility management deals with synchronizing activities, supporting main company activities by managing 
staff administration, auxiliary work activities and business environments - activities related to care of real estate, building and selecting employee administration. According to the IFMA association's definition, FM is "A method of an organization's alignment of work environment, workers, and work activities. It incorporates principles of business administration, architecture, humanities, sciences and engineering." [2]

Regarding these facts, the use of facility management (FM) can affect the ability of the organization to carry out its activities in a better way and meet all its requirements. The FM goal is mainly to provide a better working environment and condition for an organization to help it meet its main goals. FM comprises various areas, but it is facility maintenance management (FMM) that constitutes most $(65 \% \sim 85 \%)$ of the total costs incurred by FM activities. [3]

Facility maintenance is the process of increasing the utility of a building by regularly servicing capital assets, commercial appliances, and areas inside or around a building. Facilities' operations and maintenance encompasses a broad spectrum of services, competencies, processes, and tools required to assure the built environment will perform the functions for which a facility was designed and constructed [4]. But due to the lack of comprehensive models and techniques for managing a facility and the absence of adequate building maintenance policies in most companies, all maintenance activities are usually done with high cost and delay [4]. Maintenance is a process which occurs to prevent deterioration in buildings and also to repair the damages which happen in the building. Damages happen in a building when some part of it cannot functional well. There are many types of damages in a building such as in the electrical system, water supply, floor, roof, the drainage system and the walls. This problem may be solved by doing the building maintenance to repair or restore the equipment that cannot functional well. The problem occurring in a building will affect the tenants in it, and that is why the maintenance process is very important. That is, it is required to make sure the tenants are convenient and safe to use the building. The longest period in the lifecycle of a building is the operation and maintenance (O\&M) or facility management phase. [5]

The maintenance optimization problem, which has been divided into three types, namely strategy selection, maintenance planning, and maintenance scheduling, consists of the development and analysis of mathematical models to aim at improving or optimizing maintenance [6]. This article focuses on optimization scheduling maintenance.

In terms of scheduling maintenance, several models and algorithms have been proposed for optimizing scheduling in the literature. These models can be divided into two groups, namely single objective model [7] And multi objective models [8] with different objective function such as optimization model based on cost minimization [9], reliability based optimization function model [7], optimization model to safety maximization [10], risk of failure minimization model [11], maximization of profit [12], maintainability based model [13], availability based model [9]. Where different models have been applied for solving these models such as Genetic 
algorithm [14], Particle Swarm optimization [15], NSGA II [16], mixed integer linear programming [17], Monte Carlo Simulation [12].

In 2003, Cassady \& Kutanoglu proposed a new model to optimize scheduling by a Single Objective Model through considering tardiness, as the objective function, and different constraints such as maintenance time, total weight of component, usage of labor and planning period [18].

In the same year, Grall \& Roussignol proposed a new model for scheduling optimization with a Single Objective Model by considering tardiness as the objective function, and different constraints such as system availability requirements, inspection activity, usage of labor and planning period [19].

Coolen-Schrijner \& Coolen, in 2004, proposed a new model to optimize scheduling with a single objective model by considering tardiness as the objective function, and different constraints such as component age, usage of labor and planning period [1].

In 2008, Lugtigheid, Jiang \& Jardine proposed a new model for optimization planning by a single objective model by consideration of tardiness as the objective function and different constraints like maintenance time, component age, repair improvement factor and planning period [20].

Flage, Coit, Luxhoj \& Aven, in 2012, proposed a new model for optimization scheduling by a multi-objective model by consideration of tardiness as the objective function and different constraints such as component age, inspection activity, usage of labor and planning period [21].

Wang \& Zhang, in 2013, proposed a new model to optimize scheduling by a multi-objective model through considering tardiness as the objective function and different constraints like system availability requirements, component age, inspection activity, failure rate, usage of labor and planning period [22].

In 2013, Zitrou, Bedford \& Daneshkhah proposed a new model for optimizing planning by a single-objective model and the consideration of tardiness as the objective function, along with different constraints such as component age, failure rate and planning period [2].

Finkelstein, in 2015, proposed a new model to optimize planning through a multi-objective model by considering tardiness as the objective function, along with different constraints such as system availability requirements, repair improvement factor, failure rate and planning period [23].

In 2015, Lin, Huang \& Fang proposed a new model to optimize planning through a single-objective model by considering tardiness as the objective function, and different constraints such as repair improvement factor, failure rate and planning period [24].

Zhou, Li, Xi \& Lee, in 2015, proposed a new model to optimize scheduling by a single-objective model by considering tardiness as the objective function, and different constraints such as component age, repair improvement factor, failure rate, component limit, usage of labor and planning period [25].

Wang, Huang, Li \& Yang, in 2016, proposed a new model to optimize scheduling through a single-objective 
model by considering tardiness as the objective function, and different constraints such as component limit, maintenance priority, usage of labor and planning period [3].

In 2018, De Jonge \& Jakobsons proposed a new model for optimizing scheduling through a single-objective model by considering tardiness as the objective function, and different constraints as, component age, repair improvement factor, usage of labor and planning period [26].

Fouladirad, Paroissin \& Grall, in 2018, proposed a new model to optimize planning through a single-objective model by considering tardiness as the objective function, and different constraints such as component age, failure rate and planning period [27].

Based on the literature, different objective functions have been considered for the scheduling problem as indicated in Figure 1.

Figure 1.

Having perused the literature, in this paper, a new three-stage model has been presented for facility maintenance scheduling which is based on GA, PSO and the Monte Carlo Simulation model through considering different required work skills and the outsourcing possibility for each task in different locations. To answer the main question addressing the optimal number of maintenance teams and the best work flow and maintenance schedule for a whole year based on maintenance crew limitation, the current paper contributes in the following ways:

- It proposes a new three-stage model to optimize scheduling.

- It optimizes the number of crew by different skills in the first stage.

- It evaluates the fitness value for each solution through the Monte Carlo Simulation Model.

- scheduling by consideration different failure rates for different facilities in different locations

According to the above items, the remainder of this paper is organized as follows: In Section 2, the proposed model with its combination of GA, PSO and the Monte Carlo Simulation is explained. In Section 3, the proposed model is applied for a real-world case study, the results of which have been interpreted. Finally, a brief summary and some concluding notes are provided in Section 4

\section{Research methodology and proposed model}

Different model has been considered in literature to optimize maintenance scheduling problem with different assumptions, in the proposed model, the following assumptions have been considered:

- Different failure rate functions for different skills in different locations

- Out-of-work-time transferring between all locations 
- Transferring time between all locations are less than one day.

- Maintenance tasks are independent.

- There is no possibility for outsourcing.

- There are three mechanical, electrical, and simple worker skills for performing maintenance tasks.

- $\quad$ By assuming the parts' consumptions in all models, the cost of buying parts is not considered.

- The location of all crews in days 0 are the same.

- All crew can stay in all locations.

To facilitate reading, symbols related to sets, parameters, and decision variables used in the mathematical model are presented in Table 1.

Table 1.

Based on Table 1, the mathematical model to optimize the maintenance scheduling has been considered as the following equations where the objective function is based on cost minimization that consist of three parts of crew cost and transportation cost. [5]

$$
\begin{array}{lc}
\min z=\sum_{k=1}^{k} \sum_{i=1}^{n} C_{k}^{\prime} n_{k}+\sum_{j=1}^{n} \sum_{i=1}^{n} C_{i j} z_{i j}+\sum_{j=1}^{n} C_{i}^{\prime \prime} y_{i} \\
\sum_{j}^{n} S y_{i j}^{d, \mathrm{n}_{k}} \geq t_{k, i}^{d} \quad \forall k \in K, d \in D \\
Z_{i j}=\sum_{n_{k}=1}^{n} X_{i j}^{d, \mathrm{n}_{k}} \quad \forall i, \mathrm{j} \in N \\
\sum_{i=1}^{n} X_{i j}^{d, \mathrm{n}_{k}}=\sum_{i=1}^{n} X_{j i}^{d, \mathrm{n}_{k}} & \forall i, \mathrm{j} \in N, d \in D
\end{array}
$$

In this model we want to optimize the number of optimum crew by minimizing the maintenance cost for lots of facilities in different locations which each facility have its own failure by explorational probability functions for different skills. So, for solving the proposed model, a combination of GA, PSO and the Monte Carlo Simulation has been considered based on bellow pseudocode and figure 2:

1- The number of crews randomly generated as an initial generation

2- Put $\mathrm{g}=1$.

3- If $\mathrm{g}=1$ go to step 7 else go to step 4

4- Create a new generation for number of crews by updating previous generation by selection, crossover and mutation.

5- Check feasibility of each solution and refine it if necessary. 
6- Put $g=g+1$

7- Generate maintenance schedule randomly as location of particles based on number of crews.

8- Evaluate the fitness value of each solution based on Monte Carlo simulation.

9- Check the stop condition for the PSO algorithm, if it is true go to step 10 else go to step 11.

10- Check the stop condition for the model, if it is true stops the algorithm else go to step 11.

11- Determine best location of each particle

12- Determine best location of all particle

13- Update location of all particle

14- Go to step 9

Based on the proposed model, at the first stage, GA was applied to optimize the number of crew in different skills. Then, based on the results of GA, scheduling was proposed for each crew by the PSO model. Finally, the simulation model was applied to evaluate the fitness value for each scheduling.

\subsection{Genetic Algorithm}

The GA is a heuristic algorithm for random search referring to biology evolution. These algorithms have been successful in solving hard optimization problems such as TSP, VRP etc. The GA includes operations of initial population generation, fitness evaluation, selection, crossover, and mutation [28].

In this paper, GA was applied to generate the number of crew with different skills due to its flexibility and its ability to search whole parts of problem space from populations of points instead of one specific part of solutions space. So GA is an efficient and effective technique to find an approximate solution in optimization and search problems.

In proposed model, GA has been applied as bellow steps:

Step I: Randomly generate the initial population of chromosomes due to value encoding method. The $i$ 'th chromosome in the initial population is shown by $\mathrm{g}_{\mathrm{i}}{ }^{0}$ as $1 \times k$ ( $\mathrm{k}$ is the skill number), where each value in this vector is an integer number that proposes the number of crew on that skill.

$$
g_{i}^{0}=\left[\begin{array}{llll}
a_{i 1}^{0} & a_{i 2}^{0} & a_{i 3}^{0} & a_{i k}^{0}
\end{array}\right]_{1 k}
$$

All values in this vector are generated randomly. 
Step II: By considering each solution from step 1, the PSO algorithm and Monte Carlo model were run and the fitness value of each chromosome was evaluated based on result of Monte Carlo simulation model..

Step III: When the stop condition (When there has been no improvement in the population for 10 iterations) is satisfied, the GA algorithm process will be stopped and resumes in else.

Step IV: Create a new population by selection, crossover, and mutation operations.

a) Selection: Select two parent chromosomes from a population according to their fitness based on Roulette wheel selection model.

b) Crossover: in the proposed model, one point crossover has been applied, as a parameter of $r$ (integer value between 1 to $\mathrm{k}$ ) has been generated randomly and changes the row from $r$ 'th to $k$ 'th columns on parent $\mathrm{I}$ by parent $\mathrm{j}$. The result is equal to the below if $\mathrm{r}=3$.

$$
\begin{aligned}
g_{i}^{\prime 0} & =\left[\begin{array}{llll}
a_{i 1}^{0} & a_{i 2}^{0} & a_{i 3}^{0} & a_{j k}^{0}
\end{array}\right]_{1 k} \\
P_{i}^{\prime 0} & =\left[\begin{array}{llll}
a_{i 1}^{0} & a_{i 2}^{0} & a_{i 3}^{0} & a_{i k}^{0}
\end{array}\right]_{1 k}
\end{aligned}
$$

c) Mutation: in this survey, the uniform mutation model has been applied and randomly change one parent value by a random value.

$$
g_{i}^{0}=\left[\begin{array}{llll}
a_{i 1}^{0} & a_{i 2}^{0} & a_{i 3}^{0} & a_{i k}^{0}
\end{array}\right]_{1 k}
$$

Step V: Go to step II.

Based on above description, the GA pseudocode could be rewrite as bellow:

1. Randomly generate, list of solutions as a first generate,

2. Evaluation of fitness functions for each solution in the last generations.

3. Cheek stop condition, if it's true algorithm stops, else go to step 4.

4. Create a new generation by operations of crossover, mutation and selection.

5. Check feasibility of each solution and improve it, if it is required.

6. Go to steps 2 .

\subsection{PSO Algorithm}

PSO is a heuristic algorithm for finding a global optimum by iteratively Searching to increase the quality of candidate solutions. This algorithm foundation based on the group of birds to search for food in the solutions space randomly. Each bird is a single solution that moves in the solution space to look for better solutions.[29] 
The PSO has advantages and disadvantages. Advantages of the basic particle swarm optimization algorithm: PSO is based on the intelligence. It can be applied into both scientific research and engineering use. Then PSO have no overlapping and mutation calculation. The search can be carried out by the speed of the particle. During the development of several generations, only the most optimist particle can transmit information onto the other particles, and the speed of the researching is very fast. After that the calculation in PSO is very simple. Compared with the other developing calculations, it occupies the bigger optimization ability and it can be completed easily. The last one is PSO adopts the real number code, and it is decided directly by the solution. The number of the dimension is equal to the constant of the solution.[29]

In this paper, PSO was applied to optimize scheduling in the following steps: [30]

a) By considering the number of crew from GA, the initial particle location (scheduling) is randomly generated as bellow.

$$
P_{i}^{0}=\left[\begin{array}{ccc}
a_{11} & \ldots & a_{1 d} \\
\ldots & \ldots & \ldots \\
a_{n_{k} 1} & \ldots & a_{n_{k} d}
\end{array}\right] \text { where } 1 \leq a_{i j} \leq n \quad \text { for } i=1,, n_{k}, j=1 . d
$$

In this matrix $a_{i j}$ means the number of crews by $i^{\prime}$ th skill in $\mathrm{j}^{\prime}$ th location for days number $\mathrm{d}$.

b) Each solution has been defined to all maintenance request has been done.

c) Each solution has been evaluated by running the simulation model, and the fitness value of each solution has been evaluated based on the simulation model.

d) The particles' location was updated by Eq. (10) for each particle.

$$
P^{j+1}{ }_{i}=P^{j}{ }_{i}+V_{i}^{j+1}
$$

where $V_{i}^{j+1}$ is the velocity for the $i^{\prime} t h$ particles in $j+l^{\prime}$ th iteration, and is applied to the updated previous location of swarms. In Eq. (10), new location of i'th particle in iteration number $j+1$ has been changed due to value of $\mathrm{V}_{\mathrm{i}}^{\mathrm{j}+1}$ and it depended to best location of $\mathrm{i}^{\prime}$ th particle until $\mathrm{j}$ 'th iteration and best location of all particles.. The value of $\mathrm{V}$ was computed according to the formula given in Eq. (11).

$$
V_{i}^{j+1}=V_{i}^{j}+r \alpha\left[P_{\text {best }, i}^{j}-P_{i}^{j}\right]+s \beta\left[g_{\text {best }}^{j}-P_{i}^{j}\right]
$$


Based on Eq (1) the direction of i'th particle toward its best location has been evaluated with $\left[P_{\text {best }, i}^{j}-P_{i}^{j}\right]$ by the weight of $r \alpha$ and its direction toward the best value of all particles has been evaluated with $\left[g_{\text {best }}^{j}-P_{i}^{j}\right]$ by the weight of $s \beta$. In this equation, $P_{b e s t, i}^{j}$ is the best position for $i$ 'th particle to $j$ 'th iteration, $P_{b e s t}^{j}$ is the best position for all particles to $\mathrm{j}$ 'th iteration, $\alpha$ and $\beta$ are random parameters between 0 and 1 , and $\mathrm{r}$ and $\mathrm{s}$ are constants.

Based on above description, the PSO pseudocode could be rewrite as below:

1. Initial location of particles randomly generated.

2. Evaluation of fitness functions for each solution in last locations.

3. Cheek stop condition, if it's true algorithm stops, else go to step 4.

4. Cheek the best location of each particle in all iterations based on fitness function

5. Evaluation best location of all particles.

6. Update particle's locations

7. Go to steps 2 .

\subsection{Monte Carlo Simulation}

The Monte Carlo Simulation is a method to model real-world uncertainty with normal or non-normal distributions and complex performance functions. The Monte Carlo Simulation uses repeated random sampling to simulate data for a given mathematical model and evaluate the outcome [30].

The process of the Monte Carlo Simulation is based on the bellow steps:

1- Identify the transfer equation as quantitative model for the simulation.

2- Define the input parameters for the simulations.

3- Create random data for each parameter with a special probability function.

4- Simulate and analyze the result.

In this paper, the Monte Carlo Simulation was applied to calculate the fitness value of each proposed scheduling by PSO.

Fig.2.

\section{Numerical result}

In order to evaluate the proposed model's performance, it was applied for a company with 5 different locations with different facilities, where the failure function was considered as an explorational probability function with different parameters for each skill in different locations, as in Table 2, where it was redefined after each inspection. 
Table 2

The proposed model was coded on Matlab and was run for the proposed problem. The convergence trend of PSO in each loop is shown in Fig 3.

Fig.3.

Based on Fig.3, it is clear that in earlier iterations, there are large improvements in convergence in each steps, and it gets smaller in older iterations and the convergence has been achieved on $89^{\prime}$ th iteration .

In order to evaluate the proposed model's performance, it was compared with models which have been proposed by Golpira's Model. Results are given in the bellow figure.

Fig.4.

Fig.5.

Based on Figures 4 and 5, it is clear that the performance of the proposed model is better than Golpira's Model in cost and MTBF, and in some cases, the cost of the proposed model is half the total cost in Golpira's Model. In general, the cost of the proposed model in comparison with the Golpira's Model is less than $39 \%$. On the other hand, the MTBF in the proposed model is three timws of Golpira's Model in some cases, and on average, the MTBF of the proposed model compared to Golpira's Model is twice.

In order to evaluate the proposed model's performance, different types of costs in different scenarios have been shown in Fig. 6, and total cost per crews has been shown on Fig.7.

Fig.6.

Fig.7.

In order to evaluate the performance of the proposed model, we applied it in a problem with different failure rate parameters, the result of which has been shown in Fig 8.

Fig.8.

Based on Fig 8, it is clear that by the decrease of Landa in failure rate, total maintenance cost will increase. And based on Fig 9, it is clear that MTBF will increase by the increase of Landa.

Fig.9.

On the other hand, to evaluate the performance of the proposed model in different situations, Fig.10 to Fig 16 show the result of different costs of the proposed model in different situations.

Fig.10.

Fig.11. 
Fig.12.

Fig.13.

Fig.14.

Fig.15.

Fig.16

Based on the above charts, the number of crews play a key role in maintenance cost. It is true that, by increasing the number of crews in each scenario, personnel cost has been increased but it has a considerable impact on failure cost to the extent that by increasing 2 or 3 staff we are witness to great decrease in failure cost in contrast to slight increase in personnel cost. On the other hand, by increasing the number of crews more than 8 or 9 , the impacts of each increment in number of crews on failure cost is minor and they just increased the personnel cost. So, determination of the number of staff plays a key role in the cost of maintenance planning. There is a reverse relationship between the number of crews and transportation cost and it is just because of decrease in number of trips due to increasing in number of crews. So, there is a direct relationship between number of staff and personnel cost and reverse relation between the number of staff and failure cost and transportation cost.

Based on the results of the proposed model, it can be said that the model can be applied for different situations for facility with different failure rates (related to number of facilities with different ages) and different numbers of crews, where all facilities can be located in different locations. By this model, it is possible to optimize the number of crews and scheduling at the same time. The results show that the failure cost will be decreased by increasing of number of crews.

\section{Conclusion}

In this research, a new hybrid model has been presented to optimize facility maintenance scheduling for organization by owning facility with different failures rate which located in multiple location. In the proposed model the maintenance tasks have been categorized in three different groups which are independent and all maintenance action should be done with the company staff. In the proposed model the total cost including salary of crews and transportation cost should be minimized.

The main contributions of this paper include: (a) optimizing the number of crew by different skills in the first stage. (b) evaluation of fitness value for each solution through the Monte Carlo Simulation Model. (c) scheduling by consideration different failure rates for different facilities in different locations.

In order to evaluate the performance of the proposed model, it was compared with Golpira's model, and the results have shown that it is possible to reduce the cost by over $39 \%$ and reduce MTBF by over half. 


\section{Future suggestions}

For future research we proposed that:

- Solve maintenance scheduling problem by consideration of cost for materials.

- Categorized each failure in different categories by different importance.

- Consideration of outsourcing some tasks.

\section{Reference}

Coolen-Schrijner, P. and F.P. Coolen, 'Adaptive age replacement strategies based on nonparametric predictive inference'. Journal of the Operational Research Society, 2004. 55(12): p. 1281-1297. Zitrou, A., T. Bedford, and A. Daneshkhah, 'Robustness of maintenance decisions: Uncertainty modelling and value of information'. Reliability Engineering \& System Safety, 2013. 120: p. 60-71.

Liu, X., W. Wang, and R. Peng, 'An integrated production, inventory and preventive maintenance model for a multi-product production system'. Reliability Engineering \& System Safety, 2015. 137: p. 76-86.

Olanrewaju, A.L., A. Idrus, and M.F. Khamidi, 'Investigating building maintenance practices in Malaysia: a case study. Structural Survey', Journal of Industrial Mathematic, 2011.

Lavy, S. and I.M. Shohet ,'Integrated maintenance management of hospital buildings: a case study'. Construction Management and Economics, 2004. 22(1): p. 25-34. de Jonge, B. and P.A. Scarf, 'A review on maintenance optimization'. European Journal of Operational Research, 2019. Chung, S.H., F.T. Chan, and H.K. Chan, 'A modified genetic algorithm approach for scheduling of perfect maintenance in distributed production scheduling'. Engineering Applications of Artificial Intelligence, 2009. 22(7): p. 1005-1014. Abdollahzadeh, $\mathrm{H}, . \mathrm{K}$. Atashgar, and M. Abbasi, 'Multi-objective opportunistic maintenance optimization of a wind farm considering limited number of maintenance groups'. Renewable Energy, 2016. 88: p. 247-261. Wang, Y., H. Zuo, and D. Lv. 'Improved Multiobjective Maintenance Optimization of Aircraft Equipment Using Strength Pareto Genetic Algorithms with Immunity'. Fourth International Conference on Natural Computation. 2008. IEEE.

Busacca, P.G., M. Marseguerra, and E. Zio. 'Application of Genetic Algorithms to the Multi-Objective Optimization of the Inspection Times of a Safety System of a Pressurized Water Reactor'. Proceedings of the European Safety \& Reliability International Conference (ESREL'2001). 2001.

.11 Tan, Z. Li, J. Wu, Z. Zheng, J. et al., 'An evaluation of maintenance strategy using risk based inspection'. Safety science, 2011. 49(6): p. 852-860.

.12 Siddiqui, M,A. Shahid, I. Aamer, A. Jiping, L. et al., 'A Novel Idea for Optimizing Condition-Based Maintenance Using Genetic Algorithms and Continuous Event Simulation Techniques'. Mathematical Problems in Engineering, 2017. 2017.

.13 Ding, S.-H. and S. Kamaruddin, 'Maintenance policy optimization-literature review and directions'. The International Journal of Advanced Manufacturing Technology, 2015. 76(5-8): p. 1263-1283.

.14 Chikezie, C.U., A.T. Olowosulu ,and O.S. Abejide, 'Multiobjective optimization for pavement maintenance and rehabilitation programming using genetic algorithms'. Arch Appl Sci Res, 2013. 5(4): p. 76-83.

.15 Garg, H., 'Fuzzy multiobjective reliability optimization problem of industrial systems using particle swarm optimization'. Journal of Industrial Mathematics, 2013. 2013.

.16 Deb, K. and S. Karthik. 'Dynamic multi-objective optimization and decision-making using modified NSGA-II: a case study on hydro-thermal power scheduling'. International conference on evolutionary multi-criterion optimization. 2007. Springer.

.17 Riccardo, M. Riccardo, A. Teresa, Cennerazzo, Emilio. et al., 'The scheduling of maintenance. A resource-constraints mixed integer linear programming model'. Computers \& Industrial Engineering, 2015. 87: p. 561-568.

.18 Cassady, C.R. and E. Kutanoglu, 'Minimizing job tardiness using integrated preventive maintenance planning and production scheduling'. IIE transactions, 2003. 35(6): p. 503-513.

.19 L, Dieullea. C, Bérenguera. A, Gralla. M, Roussignolb., 'Sequential condition-based maintenance scheduling for a deteriorating system'. European Journal of operational research, 2003. 150(2): p. 451-461.

.20 Lugtigheid, D., X. Jiang, and A.K. Jardine, 'A finite horizon model for repairable systems with repair restrictions'. Journal of the Operational Research Society, 2008. 59(10): p. 1321-1331.

.21 Roger, F. David, W. James, T. Luxhøj, T. 'Safety constraints applied to an adaptive Bayesian condition-based maintenance optimization model'. Reliability Engineering \& System Safety, 2012. 102: p. 16-26.

.22 Wang, G.J. and Y.L. Zhang, 'Optimal repair-replacement policies for a system with two types of failures'. European Journal of Operational Research, 2013. 226(3): p. 500-506.

.23 Finkelstein, M., 'On the optimal degree of imperfect repair'. Reliability Engineering \& System Safety, 2015.138 :p. $54-58$.

.24 Lin, Z.-L., Y.-S. Huang, and C.-C. Fang, 'Non-periodic preventive maintenance with reliability thresholds for complex repairable systems'. Reliability Engineering \& System Safety, 2015. 136: p. 145-156. 
Xiaojun, Z. Yanting, L. Lifeng, X. Jay, L., 'Multi-phase preventive maintenance policy for leased equipment'. International Journal of Production Research, 2015. 53(15): p. 4528-4537.

.26 De Jonge, B. and E. Jakobsons, 'Optimizing block-based maintenance under random machine usage'. European Journal of Operational Research, 2018. 265(2): p. 703-709.

.27 Fouladirad, M., C. Paroissin, and A. Grall, 'Sensitivity of optimal replacement policies to lifetime parameter estimates'. European Journal of Operational Research, 2018. 266(3): p. 963-975.

.28 Baklacioglu, T., 'Modeling the fuel flow-rate of transport aircraft during flight phases using genetic algorithm-optimized neural networks'. Aerospace Science and Technology, 2016. 49: p. 52-62.

.29 Bai, Q., 'Analysis of particle swarm optimization algorithm'. Computer and information science, 2010. 3(1): p. 180.

.30 Gountis, V.P. and A.G. Bakirtzis, 'Bidding strategies for electricity producers in a competitive electricity marketplace'. IEEE Transactions on Power Systems, 2004. 19(1): p. 356-365. 
Faranak Zagia with master of science in civil engineering from Islamic Azad University central Tehran branch who have second grade of engineering system licenses from the Roads and Urban Ministry of Iran in fields of supervision and implementation.

Omid Motamedi Sedeh with PhD degree from Tarbiat Modares University in industrial engineer and member of national Elite Foundation of Iran who interested in fields of data mining, heuristic models and artificial intelligence. He has several papers in refereed journals and conference proceedings in different area in particular in related to deregulation power market.

Bakhtiar Ostadi is an Associate Professor of Industrial Engineering at Tarbiat Modares University. He received his PhD in Industrial Engineering from the School of Engineering at Tarbiat Modares University. In addition, He received his MSc from the University of Tehran in the Industrial Engineering and his BSc in Applied Mathematics from K.N. Toosi University of Technology. His main areas of research interests include "Data-Driven Risk Assessment", "Pricing Strategies for Bidding", "Resilience engineering”, “Quality Engineering and Management", "Process Management, Modeling and Simulation”, "Reliability Engineering", and "Customer Value, Customer Experience, and Marketing". He has published several articles in international academic journals and conferences including Int. J. Production Research, Computers \& Industrial Engineering, Energy Policy, Energy, Journal of biomedical informatics, Applied Mathematical Modelling, Journal of Statistical Computation and Simulation, Journal of Risk and Reliability, Applied Mathematics and Computation, Scientia Iranica, Iranian Journal of Management Studies, Int. J. Productivity and Quality Management, International Journal of Business Innovation and Research, etc.

Figure 1. Frequency of different objective functions in maintenance scheduling [4]

Fig.2.Proposed model

Fig.3. Convergence Rate PSO algorithm

Fig.4. Comparison of total cost in proposed model and Golpira's Model

Fig.5. Comparison of total MTBF in proposed model and Golpira's Model

Fig.6. total cost for difference cost category

Fig.7. cost per perso

.Fig.8. Total cost based on Failure rate

Fig.9. MTBF based on Failure rate

Fig.10. total cost for difference cost category in Landa=2

Fig.11. total cost for difference cost category in Landa=3

Fig.12. total cost for difference cost category in Landa $=5$

Fig.13. total cost for difference cost category in Landa $=6$

Fig.14. total cost for difference cost category in Landa $=7$

Fig.15. total cost for difference cost category in Landa $=9$

Fig. 16 total cost for difference cost category in Landa=10

Table 1. Notation of the mathematical model

Table 2. Parameters of failure rate 


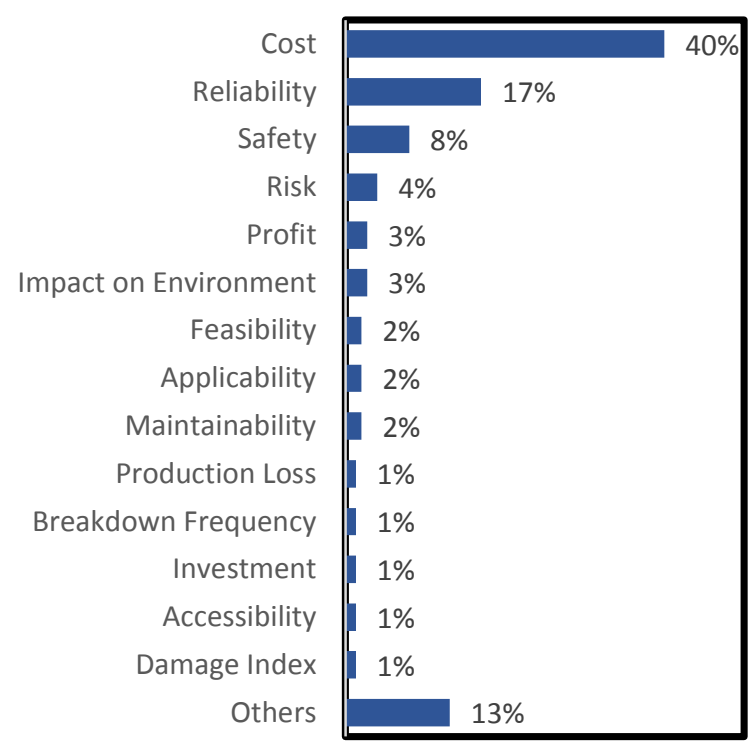

Figure 1. 


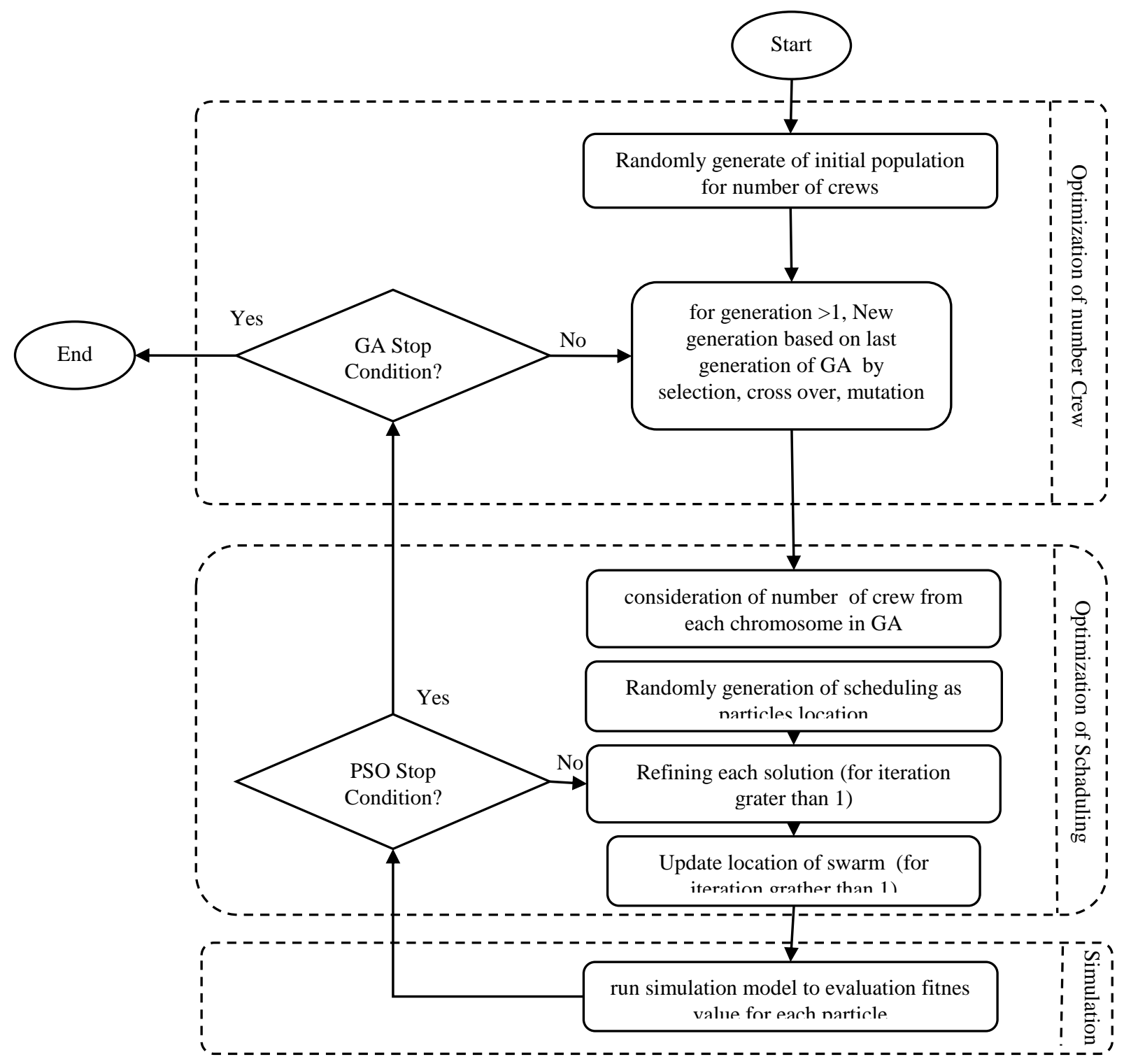

Fig.2. 


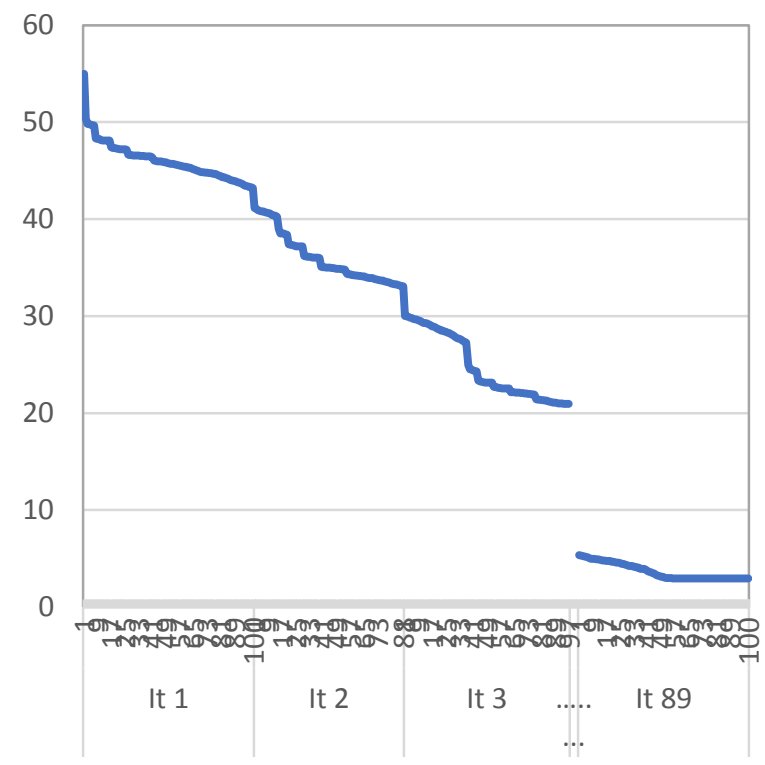

Fig.3.

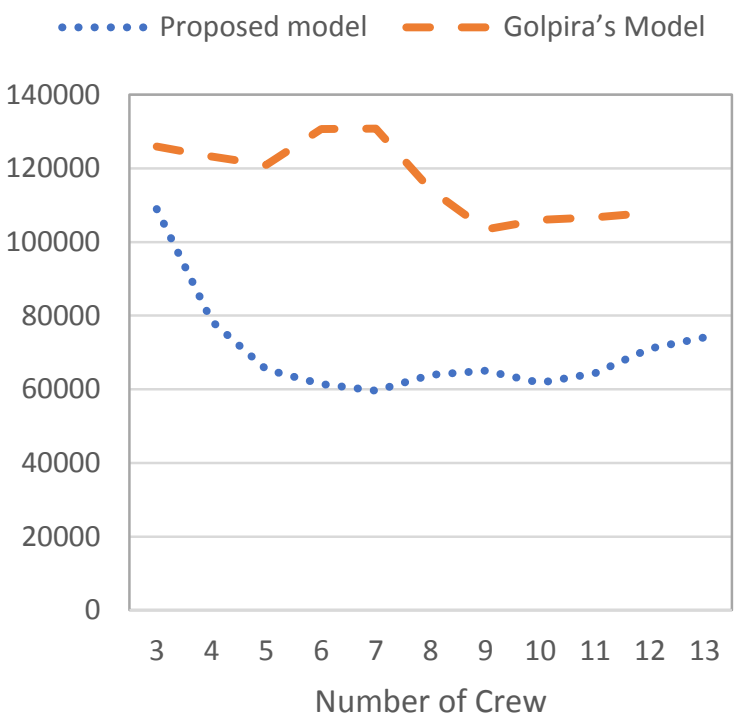

Fig.4. 


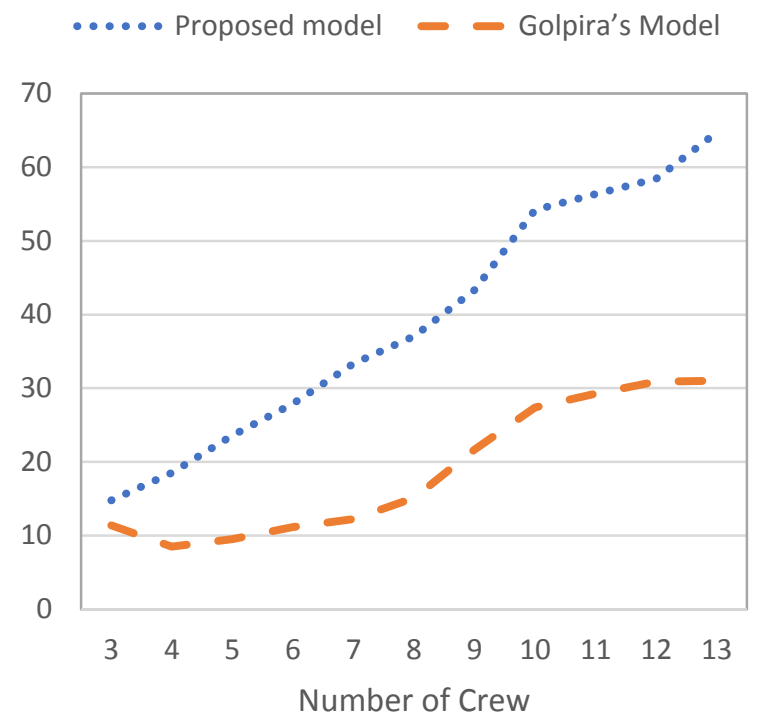

Fig.5.

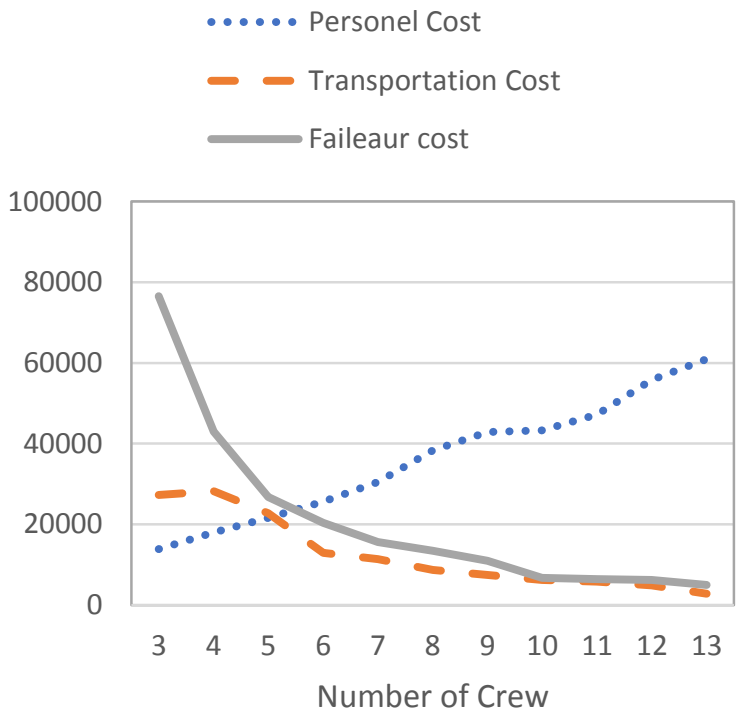

Fig.6. 


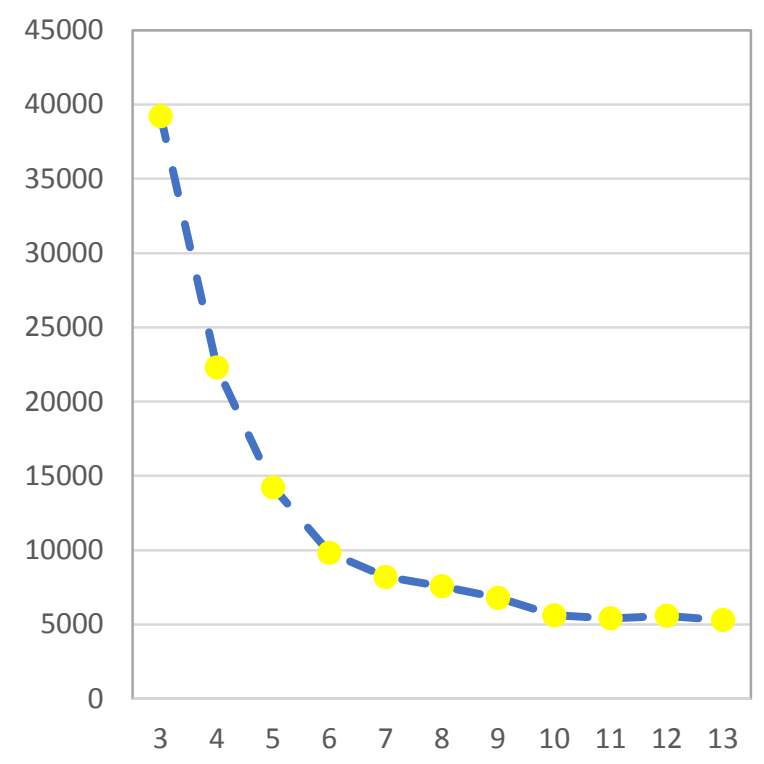

Fig.7.

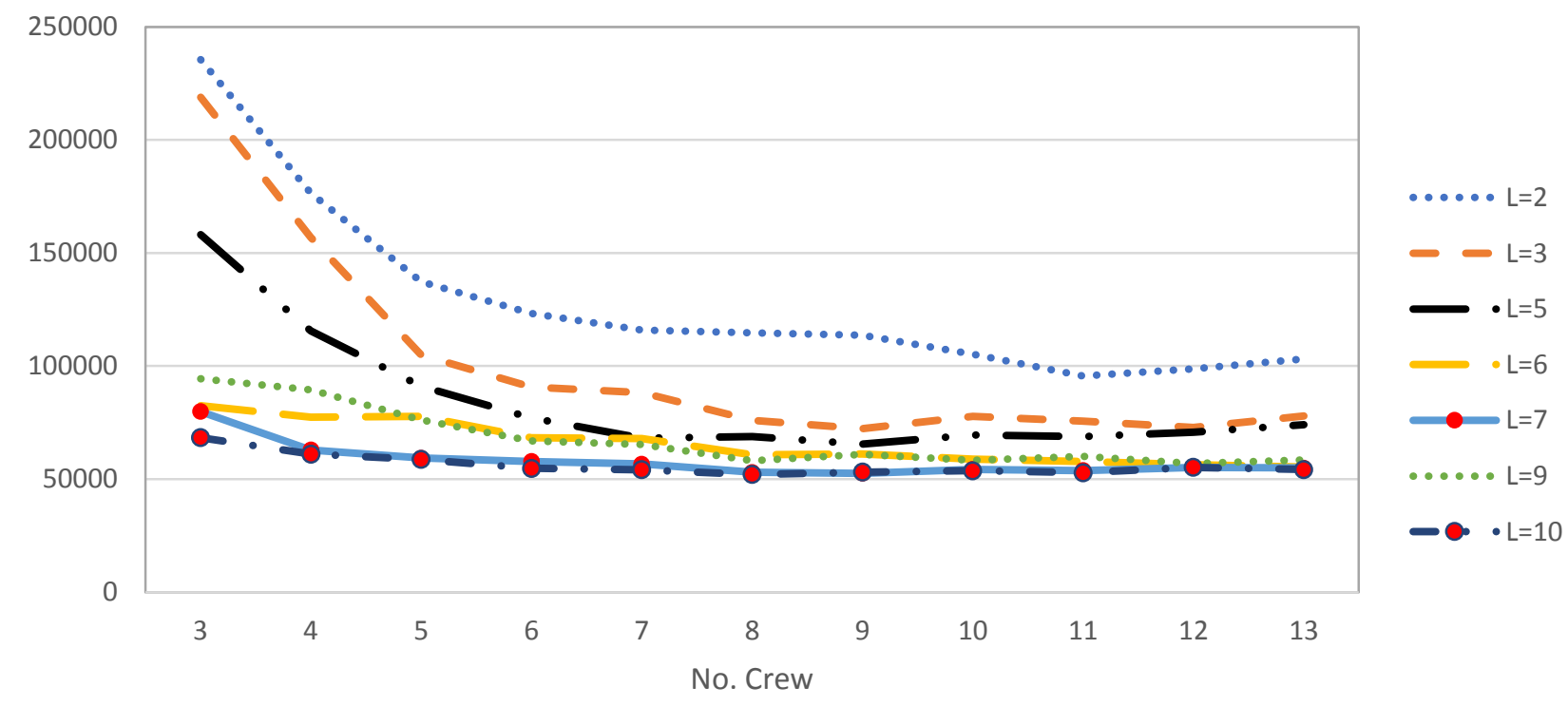

Fig.8. 


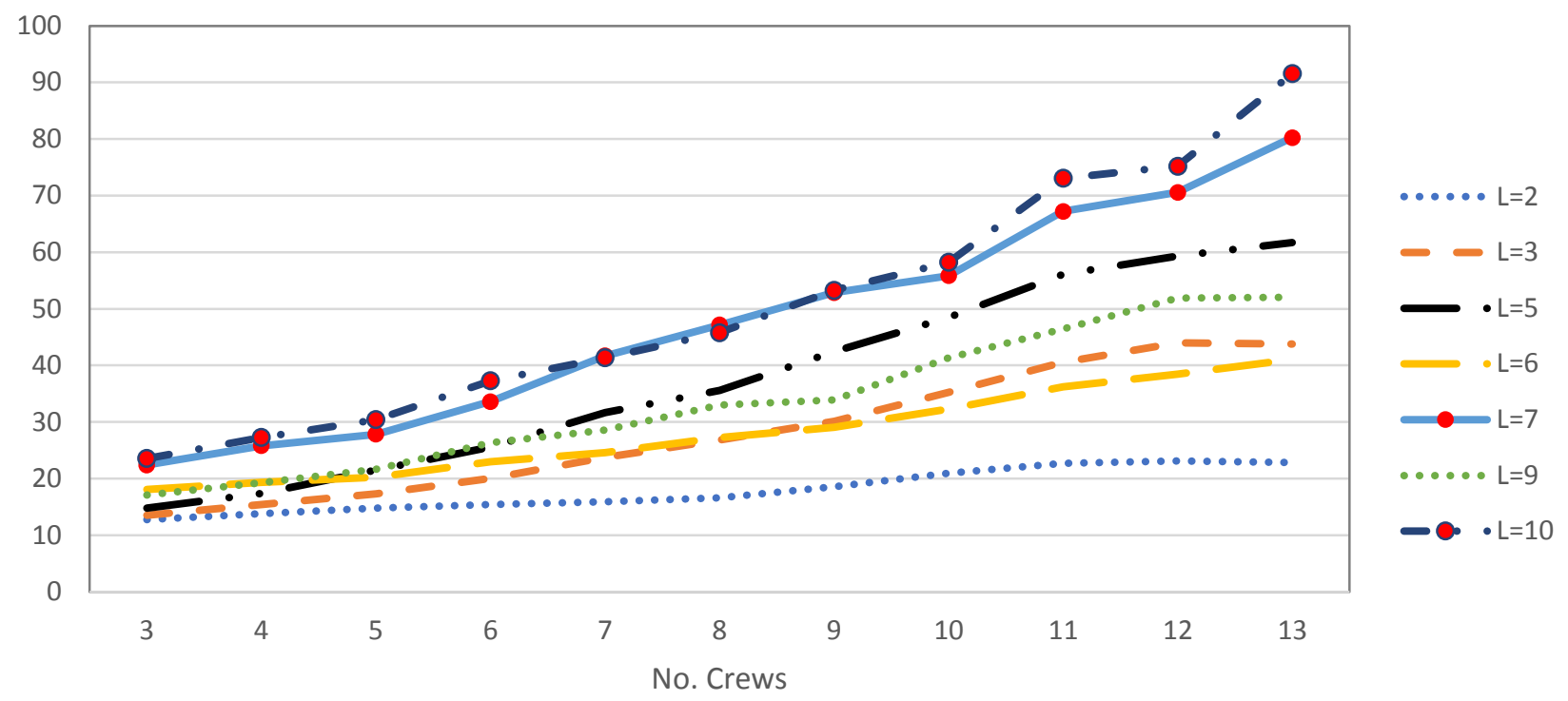

Fig.9.

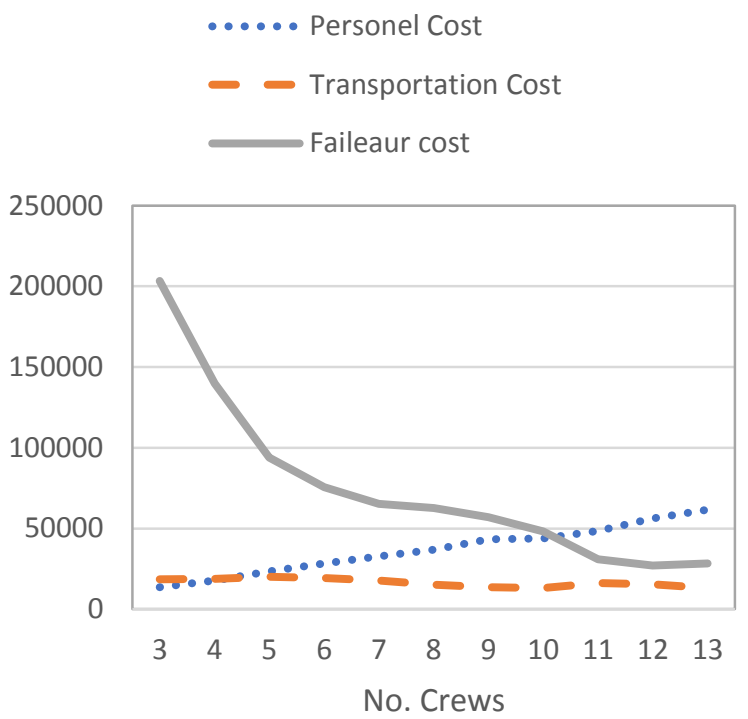

Fig.10 


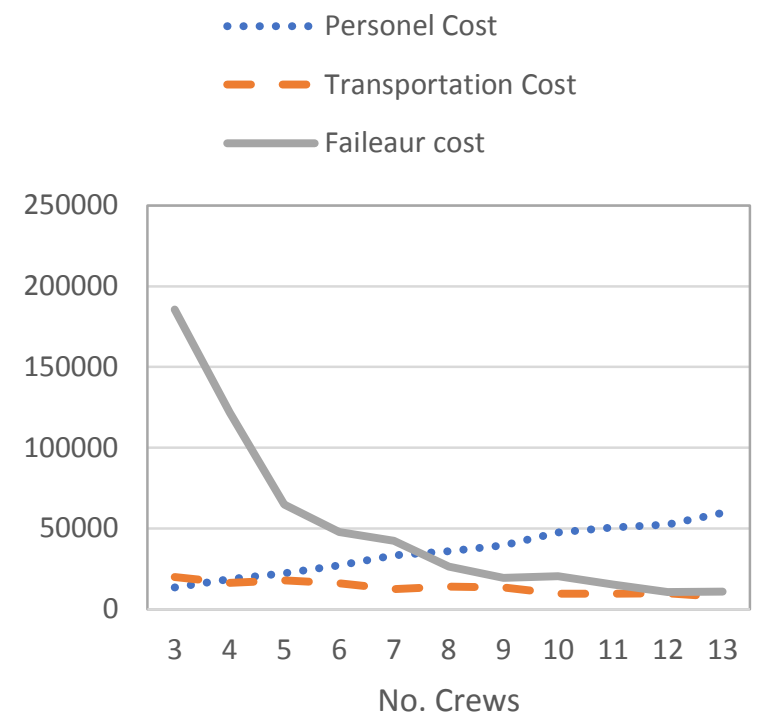

Fig.11.

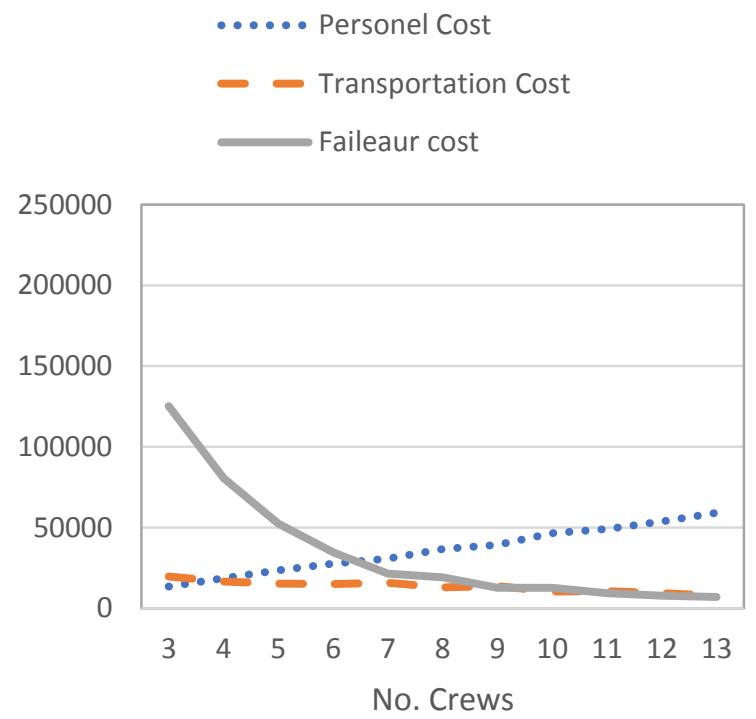

Fig.12. 


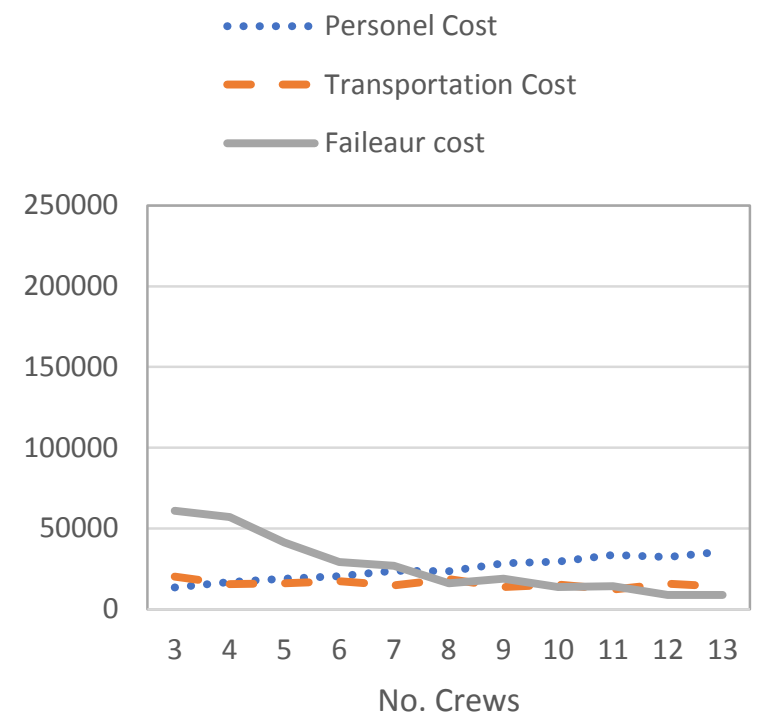

Fig.13.

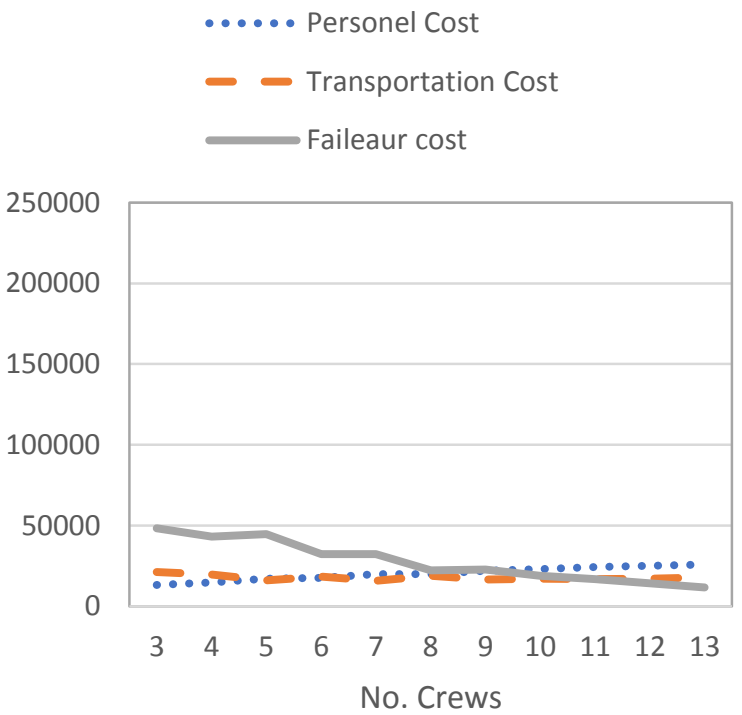

Fig.14. 


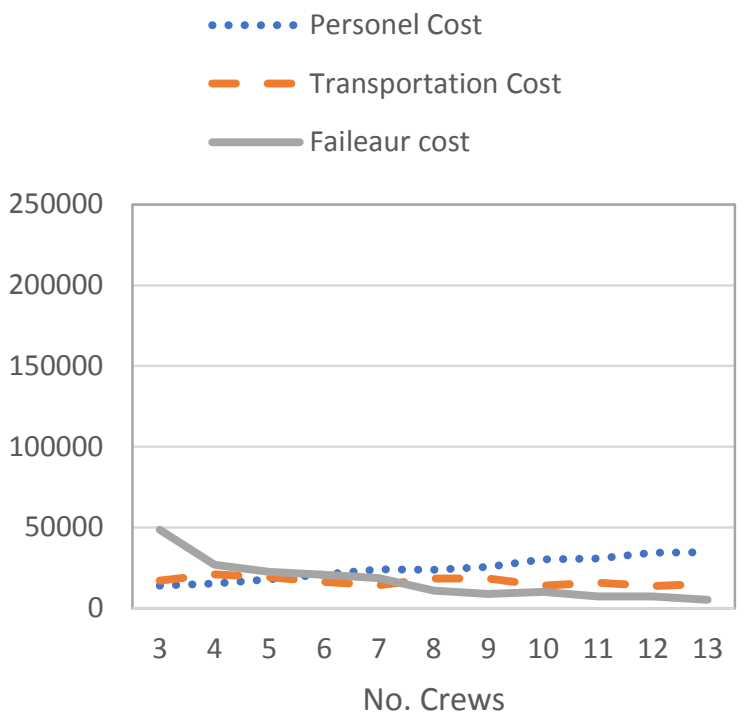

Fig.15.

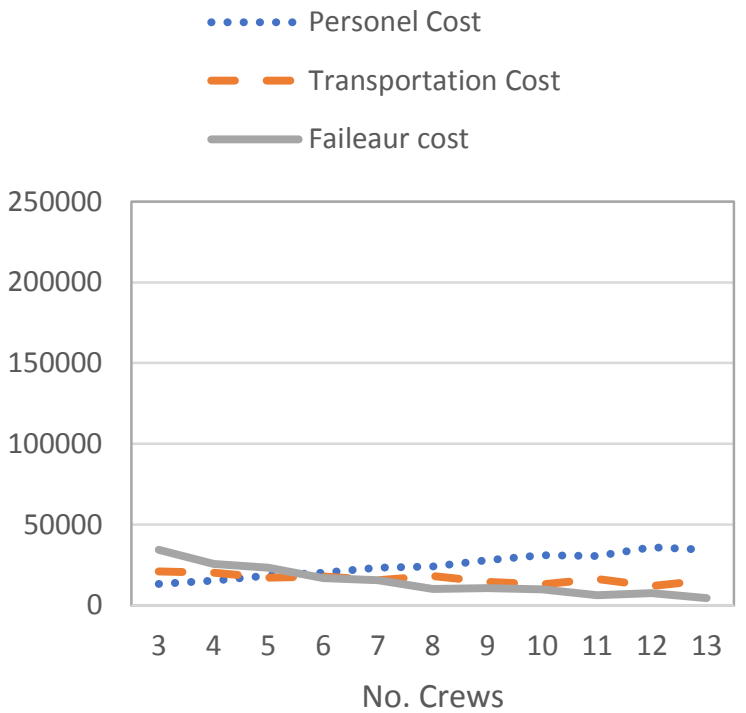

Fig.16

Table 1.

\begin{tabular}{|l|l|}
\hline Sets & \\
\hline$K=\left\{\mathrm{k}_{1}, \mathrm{k}_{2}, \mathrm{k}_{3}\right\}$ & Number of crew in each skill \\
\hline$d=\{1,2 \ldots . T\}$ & Number of days \\
\hline$n_{k}=\left\{1,2 \ldots ., n_{k}\right\}$ & Number of available workers with kth type of Speciality \\
\hline$N=\{1,2, \ldots . . n\}$ & Set of nods for services \\
\hline$A=\{(i, j) \mid i, j \in N, i \neq j\}$ & Set of arcs \\
\hline
\end{tabular}




\begin{tabular}{|l|l|}
\hline Parameters & \multicolumn{1}{|l|}{ Period of planning } \\
\hline$T$ & Period of work time in each day \\
\hline${ }^{t^{d}{ }_{k, i}}$ & The demand time of ith node for kth type of Speciality in days d \\
\hline$C_{i, j}$ & Cost of travel from nod I to nod $\mathrm{j}$ \\
\hline$C_{k}^{\prime}$ & Cost of using the kth special on all time periods \\
\hline$C_{i}^{\prime \prime}$ & Cost of failure in the i'th location \\
\hline$F_{\mathrm{k}, \mathrm{i}}$ & Failure rate on the i'th location where the kth skill is needed \\
\hline Decision Variables & \multicolumn{2}{|l}{} \\
\hline$X_{i j}^{d, n_{k}}$ & 1 if the nkth worker goes from nod $\mathrm{i}$ to nod $\mathrm{j}$ on days d and be 0 in other vice \\
\hline$X_{i j}^{\prime d, n_{k}}$ & 1 if the node i is visited by nkth worker right before node $\mathrm{j}$ on days $\mathrm{d}$ and be 0 in other vice \\
\hline$y_{i}$ & Number of failures in the i'th location \\
\hline$Z_{i j}$ & Number of transferring from nod i to nod $\mathrm{j}$ \\
\hline
\end{tabular}

Table 2.

\begin{tabular}{|l|l|l|l|}
\hline Location & Mechanical & Electrical & Simple Worker \\
\hline 1 & 5.9 & 4.1 & 3 \\
\hline 2 & 9.3 & 2.2 & 2.8 \\
\hline 3 & 7.2 & 3.1 & 4.1 \\
\hline 4 & 4.1 & 6.8 & 1.2 \\
\hline
\end{tabular}

